\title{
Strong Data Processing Constant is Achieved by Binary Inputs
}

\author{
Or Ordentlich and Yury Polyanskiy
}

\begin{abstract}
For any channel $P_{Y \mid X}$ the strong data processing constant is defined as the smallest number $\eta_{K L} \in[0,1]$ such that $I(U ; Y) \leq \eta_{K L} I(U ; X)$ holds for any Markov chain $U-X-Y$. It is shown that the value of $\eta_{K L}$ is given by that of the best binary-input subchannel of $P_{Y \mid X}$. The same result holds for any $f$-divergence, verifying a conjecture of Cohen, Kemperman and Zbaganu (1998).
\end{abstract}

Consider an arbitrary channel $P_{Y \mid X}: \mathcal{X} \rightarrow \mathcal{Y}$ with countable $\mathcal{X}$. We define the strong data processing inequality (SDPI) constant [1]

$$
\eta_{\mathrm{KL}}=\sup \frac{D\left(P_{Y \mid X} \circ P \| P_{Y \mid X} \circ Q\right)}{D(P \| Q)}
$$

where optimization is over all pairs of distributions on $\mathcal{X}$, denoted $P, Q \in \mathcal{P}(\mathcal{X})$, such that $0<D(P \| Q)<\infty$, and $P_{Y \mid X} \circ P$ is the distribution of the output $Y$ when the input $X$ is distributed according to $P \in \mathcal{P}(\mathcal{X})$. We refer to [2] for a survey of the properties and importance of the SDPI, in particular for showing equivalence to the definition in the abstract, and advertise [3] as a recent application in statistics.

When the input alphabet $\mathcal{X}$ is binary, the value of $\eta_{\mathrm{KL}}$ is relatively easy to compute, cf. [2, Appendix B]. Here we prove that for general $\mathcal{X}$ determination of $\eta_{\mathrm{KL}}$ can be reduced to the binary case.

Theorem 1: Optimization in (1) can be restricted to pairs $P, Q$ supported on two points in $\mathcal{X}$ (same for both). Proof. For two distributions $P$ and $Q$ on $\mathcal{X}$ and $\lambda \in(0,1)$ define

$$
L_{\lambda}(P, Q) \triangleq D\left(P_{Y \mid X} \circ P \| P_{Y \mid X} \circ Q\right)-\lambda D(P \| Q) .
$$

We assume that $0<D(P \| Q)<<_{\hat{Q}} \infty$ as required by the definition of $\eta_{\mathrm{KL}}$. We will show that we can find two distributions $P$ and $Q$ where $Q$ is supported on two letters in $\operatorname{supp}(Q) \triangleq\{x \in \mathcal{X}: Q(x)>0\}$, and $L_{\lambda}(\hat{P}, \hat{Q}) \geq L_{\lambda}(P, Q)$. This implies the statement, since $\eta_{\mathrm{KL}}=\sup \left\{\lambda: \sup _{P, Q} L_{\lambda}(P, Q) \geq 0\right\}$.

To that end define the convex set of distributions

$$
\begin{aligned}
& \mathcal{S} \triangleq\{\hat{Q}: \operatorname{supp}(\hat{Q}) \subseteq \operatorname{supp}(Q), \\
&\left.\sum_{x \in \operatorname{supp}(Q)} \frac{P(x)}{Q(x)} \cdot \hat{Q}(x)=1\right\} .
\end{aligned}
$$

Consider the function $g: \mathcal{S} \rightarrow \mathbb{R}$ defined as $g(\hat{Q})=L_{\lambda}\left(\frac{P}{Q} \hat{Q}, \hat{Q}\right)$. Note that $Q \in \mathcal{S}$ and $g(Q)=L_{\lambda}(P, Q)$. Consequently, $\max _{\hat{Q} \in \mathcal{S}} g(\hat{Q}) \geq L_{\lambda}(P, Q)$. Note that

$$
\hat{Q} \mapsto D\left(P_{Y \mid X} \circ \frac{P}{Q} \hat{Q} \| P_{Y \mid X} \circ \hat{Q}\right)
$$

is convex by convexity of $(P, Q) \mapsto D(P \| Q)$, and that

$$
\hat{Q} \mapsto D\left(\frac{P}{Q} \hat{Q} \| \hat{Q}\right)=\sum_{x} \hat{Q}(x) \frac{P(x)}{Q(x)} \log \frac{P(x)}{Q(x)}
$$

is linear. Thus, $\hat{Q} \mapsto g(\hat{Q})$ is convex on $\mathcal{S}$. It therefore follows that $\max _{\hat{Q} \in \mathcal{S}} g(\hat{Q})$ is obtained at an extreme point of $\mathcal{S}$. Since $\mathcal{S}$ is the intersection of the simplex with a hyperplane, its extreme points are supported on at most two atoms.

O. Ordentlich is with the Hebrew University of Jerusalem, Israel (or.ordentlich@mail.huji.ac.il). Y. Polyanskiy is with the MIT, USA (yp@mit.edu). 
Paired with [2, Appendix B] we get a corollary bounding $\eta_{\mathrm{KL}}$ in terms of the Hellinger-diameter of the channel:

$$
\begin{aligned}
\frac{1}{2} \operatorname{diam}_{\mathrm{H}^{2}}\left(P_{Y \mid X}\right) \leq \eta_{\mathrm{KL}} & \leq g\left(\frac{1}{2} \operatorname{diam}_{\mathrm{H}^{2}}\left(P_{Y \mid X}\right)\right) \\
& \leq \operatorname{diam}_{\mathrm{H}^{2}}\left(P_{Y \mid X}\right)
\end{aligned}
$$

where $g(t) \triangleq 2 t\left(1-\frac{t}{2}\right), \operatorname{diam}_{\mathrm{H}^{2}}\left(P_{Y \mid X}\right)=\sup _{x, x^{\prime}} H^{2}\left(P_{Y \mid X=x}, P_{Y \mid X=x^{\prime}}\right)$ and $H^{2}(P, Q)=2-2 \int \sqrt{d P d Q}$.

Note that the only property of divergence that we have used in the proof of Theorem 1 is convexity of $(P, Q) \mapsto$ $D(P, Q)$. This property is shared by all $f$-divergences, cf. [4]. In other words we proved:

Theorem 2: Let $\eta_{f}=\sup \frac{D_{f}\left(P_{Y \mid X} \circ P \| P_{Y \mid X} \circ Q\right)}{D_{f}(P \| Q)}$ optimized over all $P, Q \in \mathcal{P}(\mathcal{X})$ with $0<D_{f}(P, Q)<\infty$. Then the optimization can be restricted to pairs $P, Q$ supported on two common points in $\mathcal{X}$.

This fact was conjectured in [5, Open Problem 7.4].

There are two other noteworthy results that our technique entails. First, a moment of reflection confirms that we, in fact, have shown that the upper concave envelope of the set $\cup_{P_{X}, Q_{X}}\left\{\left(D_{f}\left(P_{X} \| Q_{X}\right), D_{f}\left(P_{Y} \| Q_{Y}\right)\right)\right\}$ is unchanged if we restrict the union to pairs $P_{X}, Q_{X}$ supported on two points.

Second, a similar argument holds for the post-SDPI coefficient of a channel [6], defined as

$$
\eta_{K L}^{(p)}\left(P_{Y \mid X}\right)=\inf \{\eta: I(U ; X) \leq \eta I(U ; Y) \quad \forall X-Y-U\} .
$$

Namely, we have that $\eta_{K L}^{(p)}$ can be computed by restricting $X$ to take two values. Indeed, fix an arbitrary $P_{X, Y, U}$ s.t. $X-Y-U$. As shown in [2, Theorem 4] one can safely assume $U$ to be binary. Now, consider a set $\mathcal{S}$ of all $\hat{P}_{X}$ such that the joint distribution $\hat{P}_{X, Y, U}=\hat{P}_{X} P_{Y \mid X} P_{U \mid Y}$ satisfies $\hat{P}_{U}=P_{U}$. Since $U$ is binary, $\mathcal{S}$ is an intersection of a hyperplane with a simplex. Now, the function $\hat{P}_{X} \mapsto \hat{I}(U ; X)-\lambda \hat{I}(U ; Y)$ is linear in $\hat{P}_{X}$ over $\mathcal{S}$. Consequently, the maximum (and the minimum) of this function is attained at a binary $\hat{P}_{X}$.

\section{REFERENCES}

[1] R. Ahlswede and P. Gács, "Spreading of sets in product spaces and hypercontraction of the markov operator," The annals of probability, pp. 925-939, 1976.

[2] Y. Polyanskiy and Y. Wu, "Strong data-processing inequalities for channels and Bayesian networks," in Convexity and Concentration. Springer, 2017, pp. 211-249.

[3] _ "Application of the information-percolation method to reconstruction problems on graphs," Mathematical Statistics and Learning, vol. 2, no. 1, pp. 1-24, 2020.

[4] I. Csiszár, "Information-type measures of difference of probability distributions and indirect observation," studia scientiarum Mathematicarum Hungarica, vol. 2, pp. 229-318, 1967.

[5] J. Cohen, J. H. Kempermann, and G. Zbaganu, Comparisons of stochastic matrices with applications in information theory, statistics, economics and population. Springer Science \& Business Media, 1998.

[6] Y. Polyanskiy, "Post-SDPI and distributed estimation", Lecture 5, Information-Theoretic Methods in Statistics and Computer Science, EPFL, 2019. http://people.lids.mit.edu/yp/homepage/data/LN_sdpi3.pdf 